Conclusion Overall, patients appreciate honest, personalized and attentive communication on a limited life expectancy enabling reflections and communications about ACP.

\section{OP67 PATIENT PERSPECTIVES ON INFORMATION PROVISION AND ADVANCE CARE PLANNING REGARDING IMPLANTABLE CARDIOVERTER DEFIBRILLATOR DEACTIVATION AT THE END OF LIFE}

R Stoevelaar*, A Brinkman-Stoppelenburg, R Bhagwandien, R Van Bruchem-Visser, D Theuns, A van der Heide, J Rietjens. Erasmus MC, Rotterdam, Netherlands

\subsection{6/spcare-2019-ACPICONGRESSABS.67}

Background Implantable Cardioverter Defibrillator (ICD) shocks can negatively influence the last phase of life. Advance care planning, timely and frequently discussing ICD deactivation with the patient, can help patients to make a wellinformed decision about future ICD deactivation. Perspectives of patients on ICD deactivation are however largely unknown. Methods Focus groups with ICD patients were conducted using a predefined topic list and were audiotaped and transcribed. Transcripts were analyzed using the constant comparative method.

Results Forty-one patients participated in a total of five focus groups. Average age was 64 years and $56 \%$ of patients were male. Many patients expressed a need for more information about ICD deactivation. Although most patients agreed that it would be appropriate to deactivate the ICD on a certain point in the disease trajectory, they had difficulties deciding on the right moment to do so, indicating the need to frequently re-assess their preferences. Possible circumstances in which patients would consider deactivating their ICD in order to avoid shocks were: a terminal stage of their disease, and a diminished quality of life. Arguments against deactivation were also mentioned, and mainly concerned not wanting to give up on life, both for themselves and for their family.

Conclusion(s) There is room for improvement in informing patients about ICD deactivation in the last phase of life. We found that patients' perspectives towards ICD deactivation were highly personal and sometimes ambivalent. This emphasizes the importance of early and recurring discussions on this topic.

\section{OP68 A CLUSTER-RANDOMIZED TRIAL OF A NURSE-LED ADVANCE CARE PLANNING SESSION IN PATIENTS WITH COPD AND THEIR LOVED ONES}

${ }^{1} \mathrm{C}$ Houben*, ${ }^{1} \mathrm{M}$ Spruit, ${ }^{2} \mathrm{H}$ Luyten, ${ }^{3} \mathrm{H}$ Pennings, ${ }^{4} \mathrm{~V}$ van den Boogaart, ${ }^{5} \mathrm{~J}$ Creemers, ${ }^{6} \mathrm{G}$ Wesseling, ${ }^{6} \mathrm{E}$ Wouters, ${ }^{1} \mathrm{D}$ Janssen. ${ }^{1} \mathrm{C}$ iro, Horn, Netherlands; ${ }^{2}$ University of Twente, Enschede, Netherlands; ${ }^{3}$ St Laurentius Hospital, Roermond, Netherlands; ${ }^{4}$ VieCuri Medical Centre, Venlo, Netherlands; ${ }^{5}$ Catharina Hospital Eindhoven, Eindhoven, Netherlands; ${ }^{6}$ Maastricht UMC+, Maastricht, Netherlands

\subsection{6/spcare-2019-ACPICONGRESSABS.68}

Background Advance Care Planning (ACP) is uncommon in patients with COPD.

Aims To assess whether a nurse-led ACP-intervention can improve quality of patient-physician end-of-life care communication in patients with COPD. Furthermore, the influence of an ACP-intervention on symptoms of anxiety and depression in patients and loved ones was studied.
Methods A multicenter cluster randomized-controlled trial (RCT) in patients with advanced COPD was performed. The intervention group received an 1.5-hours structured nurse-led ACP-session. Outcomes were: quality of patient-physician endof-life care communication, prevalence of ACP-discussions six months after baseline, and symptoms of anxiety and depression in patients and loved ones.

Results 165 patients were enrolled (89 intervention: mean age 65.7 (9.2) years; 49.4\% male; 76 control: mean age 69.5 (9.0) years; $57.9 \%$ male). The improvement of quality of patient-physician end-of-life care communication was significantly higher in the intervention group compared to the control group (<.001). The ACP-intervention was significantly associated with the occurrence of an ACP-discussion with physicians within 6 months $(\mathrm{p}=.003)$. At follow-up, symptoms of anxiety were significantly lower in loved ones in the intervention group compared to the control group $(p=.02)$. Symptoms of anxiety in patients, and symptoms of depression in both patients and loved ones were comparable at follow-up ( $\mathrm{p}>.05)$.

Conclusion One nurse-led ACP-intervention session improves patient-physician end-of-life care communication without causing psychosocial distress in both patients and loved ones.

The Advance Care Planning study is supported by Lung Foundation Netherlands.

\section{OP69 EFFECTIVENESS OF ADVANCE CARE PLANNING IN IMPROVING END OF LIFE CARE FOR PATIENTS WITH ADVANCED HEART FAILURE}

C Malhotra*, D Sim, F Jaufeerally, N Nadkarni, H Meibo, E Finkelstein. Duke-NUS Medical School, Singapore, Singapore

\subsection{6/spcare-2019-ACPICONGRESSABS.69}

Background Our primary aim was to assess, among advanced heart failure patients, effectiveness of Advance care planning (ACP) in ensuring end of life (EOL) care consistent with patient wishes. Secondary aims were to assess its impact on patients' decisional conflict, discussion of care preferences with surrogates, illness understanding, anxiety, depression and quality of life.

Methods We conducted a randomized controlled trial of ACP (based on Respecting Choices Model) versus usual care in Singapore. 282 patients hospitalized with heart failure and NYHA III and IV symptoms were randomized to ACP (93) or control (189) arm. They answered up to 6 followup surveys conducted every 4 months. Primary outcome was assessed in the deceased sample $(89 ; 23$ in ACP, 66 in control arm). Both intention-to-treat and per-protocol analyses were done.

Results 63\% of ACP arm received intervention. Deceased patients in ACP arm were no more likely to have their wishes followed for EOL treatments (35\% in ACP vs $44 \%$ in control; $\mathrm{p}=0.47$ ) but were more likely to have their wishes followed for cardiopulmonary resuscitation $(83 \%$ in ACP vs $62 \%$ in control, $p=0.12$ ) though the difference was not statistically significant. At first follow-up, ACP patients had lower decisional conflict $(\beta=-10.8, p<0.01)$ and were more likely to discuss preferences with their surrogate decision maker $(\beta=1.3, p=0.04)$. Both arms did not differ on other patient outcomes. Per-protocol analyses showed similar results. 
Conclusion ACP has benefits in improving decision making. More needs to be done to improve implementation of ACP for patients with heart failure.

\section{OP70 NORMATIVE PRINCIPLES OF ADVANCE CARE PLANNING: A SYSTEMATIC LITERATURE REVIEW}

${ }^{1} \mathrm{~N}$ Fleuren*, ${ }^{1} \mathrm{M}$ Depla, ${ }^{2} \mathrm{D}$ Janssen, ${ }^{1} \mathrm{M}$ Huisman, ${ }^{1} \mathrm{C}$ Hertogh. ${ }^{1}$ Amsterdam UMC, Vrije Universiteit Amsterdam, Amsterdam Public Health, Amsterdam, Netherlands: ${ }^{2} \mathrm{MUMC}+$ Maastricht, Netherlands

\subsection{6/spcare-2019-ACPICONGRESSABS.70}

Background Since the introduction of the concept of advance care planning (ACP), many studies have been conducted exploring beneficial effects. These studies show a heterogeneity in clinical endpoints, which reflects diversity of goals connected to ACP. Clarification of underlying normative principles of ACP is crucial in understanding both motivation and hesitation to initiate ACP among health care professionals and patients. This study aims to clarify normative principles of ACP and to get insight in the range of normative principles that comprise the legitimacy of ACP.

Methods Systematic literature search in PubMed, EMBASE, PsychInfo, CINAHL and Cochrane Library, using various search terms for 'ACP' and 'ethics'. Articles on normative aspects of ACP were included, based on title and abstract. Due to the quantity of inclusions, of which many had similar content, purposive sampling was used to select articles for full text document analysis. Analysis stopped once saturation was reached. Sensitivity analysis was performed to guarantee that unfrequently mentioned goals and objections were found as well.

Results In total, 6497 unique articles were found of which 183 were included. Saturation was reached after document analysis of 55 articles (30\%); this yielded 211 codes concerning normative principles of ACP. We identified 5 main normative principles for ACP: respecting individual patient autonomy, improving quality of care, strengthening relationships, improving quality of life, and reducing overtreatment. Conclusion Defining normative principles of ACP should serve as a starting point when developing ACP interventions and selecting outcome measures to evaluate ACP interventions.

\section{OP71 ACP ALBERTA: COLLABORATIVE ACTION OF HEALTH CARE, LEGAL, GOVERNMENT, COMMUNITY AND ACADEMIC SECTORS TO INCREASE PARTICIPATION IN ADVANCE CARE PLANNING}

${ }^{1} \mathrm{M}$ Douglas*, ${ }^{2} \mathrm{~K}$ Fassbender, ${ }^{3} \mathrm{~N}$ Ries, ${ }^{4} \mathrm{E}$ Wasylenko, ${ }^{5} \mathrm{P}$ Biondo, ${ }^{5} \mathrm{~J}$ Simon, ${ }^{5} \mathrm{~N}$ Hagen, ${ }^{6}$ BS Chamaschuk. ' University of Alberta, Edmonton, Canada; ${ }^{3}$ University of Technology Sydney, Sydney, Australia; ${ }^{4}$ Health Quality Council of Alberta, Calgary, Canada; ${ }^{5}$ University of Calgary, Calgary, Canada; ${ }^{6}$ Reynolds Mirth Richards and Farmer, Edmonton, Canada

\subsection{6/spcare-2019-ACPICONGRESSABS.71}

Background In 2014, Alberta's health service providers implemented a province-wide Advance Care Planning (ACP) and Goals of Care Designation policy. Despite significant efforts, barriers to full implementation remain including lack of public comprehension across health, legal and other public systems. A World Café consultation revealed multi jurisdictional recommendations to 'normalize ACP.'
Methods Through the ACP Collaborative Research Innovation Opportunities program, we formed a collaboration with lawyers, Legal Education Society of Alberta, Canadian Bar Association, Law Society of Alberta, palliative care physicians, patient advocates, academics, provincial health care providers and other stakeholders. A community of practice propagated spontaneously. Surveys and focus groups identified barriers, knowledge and resource gaps, and novel solutions, including joint health-legal education.

Results Traditional approaches to ACP have been siloed. We expand on the innovative medico-legal framework to include other stakeholders including community agencies, faith groups, health advocacy agencies, national ACP projects, and the financial planning and insurance industries. Ongoing collaborative activities include community engagement, education, policy and practice innovation, which bundles activities relating to health care planning with those relating to personal and financial planning. Our business case addresses the identified gaps and adopts public recommendations through activities to coordinate, integrate and/or support development/implementation of a community volunteer program, an electronic registry for ACP and Goals of Care documentation, and a legal toolkit.

Conclusion In practice, ACP spans medical, legal, social and personal domains. Multi-disciplinary and multi-sector approaches are posited to improve knowledge and uptake of ACP while improving the quality of life of Albertans.

\section{OP72 WHO IS IN CONTROL? CULTURAL AND INSTITUTIONAL BARRIERS TO EOL DECISION IN JAPAN AND THE UNITED STATES}

A Morita*. Hospice Hawaii, Honolulu, USA

\subsection{6/spcare-2019-ACPICONGRESSABS.72}

Japanese and American healthcare providers enjoy abundant medical resources at their disposal, yet both systems often fail to adequately address a patient's end-of-life (EOL) wishes for vastly different reasons.

In a society as highly structured in hierarchical and patriarchal dimensions as Japan's, a terminal patient's wishes are often superseded by the treating physician's suggestions in deference to the doctor's education and social status. In addition, a family member who has cultural authority tends to speak and make decisions on behalf of the dying person and the family.

Similarly, American healthcare providers often avoid the topic of EOL decision-making to deflect away from the realization that in spite of their formidable arsenal of available medical treatments, they are powerless to help. The American system of medical training also encourages physicians to advise their patients to fight an incurable disease to the end because the hope of prolonging life is never lost.

These cultural versus institutional factors have the same result. Oftentimes, the EOL wishes of a terminal patient is either not heard or lost at best. In either case, these factors have a major detrimental impact on the quality of a patient's end-of-life experience as their wishes fall prey to more dominant cultural or social factors. This theoretical study discusses the importance of EOL conversation in providing quality care, and discusses how the medical professions in both countries 